\title{
Movement behaviour determines competitive outcome and spread rates in strongly heterogeneous landscapes
}

\author{
Gabriel Andreguetto Maciel ${ }^{1,3}$ (D) . Frithjof Lutscher ${ }^{2}$
}

Received: 7 September 2017 / Accepted: 12 February 2018 / Published online: 10 March 2018

(C) Springer Science+Business Media B.V., part of Springer Nature 2018

\begin{abstract}
Classical models for biological invasions were single-species models in homogeneous landscapes, but most invasions happen in the presence of interacting species and in heterogeneous environments. The combination of spatial variation and species interaction could alter the spreading process significantly. For example, the "environmental heterogeneity hypothesis of invasions' posits that heterogeneity offers more opportunities for invaders and reduces the negative impact on native species. Environmental heterogeneity offers an obvious coexistence mechanism on the regional scale if two or more competing species have different spatial niches, i.e. if the local competitive advantage changes in space. We consider a more subtle mechanism of space use through individual movement behaviour when the local competitive advantage remains with the same species. Specifically, we model the densities of two species, diffusing and competing in an infinite landscape consisting of two types of patches. We include individual behaviour in terms of movement rate and patch preference. We consider the scenario that one of the species is the stronger local competitor in both patch types. We then uncover a number of mechanisms-based solely on movement behaviour-through which these two species can coexist regionally, how the inferior competitor can replace the superior competitor globally, or how a bistable situation can arise between the two. We calculate mutual invasion conditions as well as mutual spatial spread rates, and we show that spread rates may depend on movement parameters in unexpected ways.
\end{abstract}

Keywords Competition · Reaction-diffusion equations · Movement behaviour $\cdot$ Patchy landscapes $\cdot$ Homogenization

\section{Introduction}

Biological invasions of alien species are a widespread phenomenon that can pose a major threat for native species and is believed to be a major cause of global decline of regional biodiversity (Tobin et al. 2011). Understanding the circumstances under which an alien species can successfully establish and the speed with which it can spread into a new environment is a focal point in ecological research (Hastings

Gabriel Andreguetto Maciel

gabriel.andreguetto@gmail.com

1 Department of Mathematics and Statistics, University of Ottawa, Ottawa, Canada

2 Department of Mathematics and Statistics and Department of Biology, University of Ottawa, Ottawa, Canada

3 Present address: Instituto de Física Teórica, São Paulo State University (UNESP), R. Dr. Teobaldo Ferraz 271, São Paulo, 01140-070, Brazil et al. 2005; Melbourne et al. 2007). Ever since the pioneering work by Fisher (1937) and Skellam (1951), reactiondiffusion equations have been the modelling framework of choice for biological invasions. In the simplest case, the density of the invading species, $u(x, t)$, with $x \in \mathbb{R}$ and $t>0$ satisfies the equation

$$
\frac{\partial u}{\partial t}=d \frac{\partial^{2} u}{\partial x^{2}}+r u(1-a u)
$$

Parameter $d$ is the diffusion coefficient, $r$ stands for the maximum per-capita growth rate, and $a$ indicates the intraspecific competition coefficient. The long-term behaviour of solutions of (1) is well understood: a locally introduced population will spread at an asymptotic speed of $c^{*}=$ $2 \sqrt{d r}$, and monotone travelling wave solutions exist for all wave speeds $c \geq c^{*}$ (Weinberger 1982).

As habitats become increasingly fragmented, mainly due to human activities, invasion processes often occur in environments composed of mosaics of patches with different characteristics. Shigesada et al. (1986) extended the model in (1) to include spatial heterogeneity in the 
form of periodically alternating patches of two different characteristics ('good' and 'bad'). We will refer to such landscapes as 'patchy'. Weinberger (2002) proved that, under some conditions, the same spread speed results hold as for the homogeneous landscape: there exists an asymptotic spreading speed, and there exists a travelling periodic wave for all speeds larger than this spreading speed. There is no simple explicit formula for the spreading speed in a heterogeneous landscape, but an implicit expression can be calculated for a patchy landscape (Shigesada et al. 1986).

Many empirical studies document individual movement bias towards favourable habitat patches (Schultz and Crone 2001; Schtickzelle and Baguette 2003; Crone and Schultz 2008), as well as patch-specific movement rates (Reeve et al. 2008). Maciel and Lutscher (2013) extended the original model by Shigesada et al. (1986) and included individual movement preference at the interface between two patch types in the equations. They calculated persistence conditions and the minimal speed of a travelling periodic wave. They found that the individual-level processes of movement and habitat preference crucially affect spread rates in heterogeneous landscapes.

Most biological invasions occur not in isolation but rather in the presence of and interaction with resident species. These interactions need to be included in the description of the invasion process to assess the effects of invasions on native communities and on biodiversity. In this work, we will extend our previous model and analysis to the case of two competing species. We focus on competition, since it is known to potentially slow, halt, or reverse an invasion; see Lewis et al. (2016) and references therein.

A classical example is the spread of the North American grey squirrel (Sciurus carolinensis) and its resident competitor, the native red squirrel (Sciurus vulgaris) in Britain in the early 1900s (Okubo et al. 1989). Following the invasion, red squirrels were completely eliminated from some locations. Several hypotheses for the cause of these local extinctions were put forward, but the negative competitive effect on the red squirrel by the grey is generally accepted (see Okubo et al. 1989; Gurnell et al. 2004 and references therein). Interestingly, in North America, the niches for the grey squirrel (mixed hardwood) and red squirrel (conifer) are different. In Britain, however, red squirrels historically thrived in both types so that there is much less niche separation. Competition between the two species is through various direct and indirect mechanisms (again, see Okubo et al. (1989) and references therein for details). In strongly heterogeneous landscapes, squirrel movement exhibits the characteristics mentioned above: patch preference and movement bias at (but not only at) interfaces (Bakker and van Vuren 2004; Haughand and Larsen 2004).
Okubo et al. (1989) extended (1) to two species in a homogeneous landscape and included competition of Lotka-Volterra type, which led them to study the system

$$
\begin{aligned}
& \frac{\partial u_{1}}{\partial t}=d_{1} \frac{\partial^{2} u_{1}}{\partial x^{2}}+r_{1} u_{1}\left(1-u_{1}-a_{1} u_{2}\right), \\
& \frac{\partial u_{2}}{\partial t}=d_{2} \frac{\partial^{2} u_{2}}{\partial x^{2}}+r_{2} u_{2}\left(1-u_{2}-a_{2} u_{1}\right) .
\end{aligned}
$$

Here, the densities of the two species are denoted by $u_{1,2}$, their respective diffusion coefficients and maximum growth rates are $d_{1,2}$ and $r_{1,2}$, whereas the intra-specific competition coefficients have been scaled to unity. Parameters $a_{i}$ denote the effects of inter-specific competition on species $i$. All parameters are assumed positive.

The outcome of competition in the non-spatial LotkaVolterra model (i.e. when $d_{1,2}=0$ ) is well understood: If $a_{1,2}<1$, the two species will coexist. If $a_{1}<1<a_{2}$, species 1 will outcompete and replace species 2 , whereas if $a_{1,2}>1$, both single-species equilibria are locally stable. In the latter case, the outcome of competition depends on initial conditions, a situation sometimes termed 'bistable' or 'founder control'. For the spatial model (i.e. when $d_{1,2}>0$ ), determining the speed at which one species may spread in the presence of the other is more subtle (Hosono 1998). Lewis et al. (2002) considered the scenario that an 'invader' species 1 is introduced into an initially homogeneously distributed (at carrying capacity) 'resident' species 2 . They proved that under some sufficient, but not necessary conditions, the spreading speed of the invader is 'linearly determined' (van den Bosch et al. 1992). This means that in practice, the speed can be calculated by linearizing the equation for the invader at the resident-only steady state $\left(0, u_{2}^{*}\right)=(0,1)$. This linearized equation reads

$\frac{\partial u_{1}}{\partial t}=d_{1} \frac{\partial^{2} u_{1}}{\partial x^{2}}+r_{1} u_{1}\left(1-a_{1}\right)$.

Since this equation has the same form as model (1), the corresponding formula for $c^{*}$ is

$c^{*}=2 \sqrt{d_{1} r_{1}\left(1-a_{1}\right)}$,

provided $a_{1}<1$. The sufficient conditions for this reasoning to hold are

$$
\begin{aligned}
& r_{1}\left(1-a_{1}\right)>0, \quad r_{2} a_{2}>0, \quad d_{2} / d_{1} \leq 2, \quad \text { and } \\
& \left(a_{2} a_{1}-1\right) /\left(1-a_{1}\right) \leq\left(2-d_{2} / d_{1}\right) r_{1} / r_{2} .
\end{aligned}
$$

If $a_{1}>1$, then species 1 cannot invade.

One can interchange the roles of resident and invader to arrive at mutual invasion conditions and speeds. If $a_{1}<1<$ $a_{2}$, species 1 will eventually replace species 2 , whereas if $a_{1,2}<1$ then both species will eventually coexist. We use these results in our analysis below. 
In homogeneous landscapes, competing species can only coexist under the assumption that inter-specific competition is weak, yet coexistence is ubiquitous in nature. Habitat heterogeneity has been shown to promote coexistence in several studies (Cantrell et al. 2007). The question of invasions of competitors, and in particular the speed of invasion in heterogeneous landscapes, is studied rarely and under limited assumptions, e.g. by Cruywagen et al. (1996) for reaction-diffusion equations and Samia and Lutscher (2010) for integrodifference equations. In particular, it is not known how habitat-specific dispersal behaviour and habitat preference (see above) affect the outcome of the competition process and the speed at which invasions progress. While it is obvious that individual movement strategies offer a number of mechanisms that impact competitive outcome and spreading speed in patchy landscapes, the details are unclear. The exploration of these mechanisms is the main goal of our work.

Melbourne et al. (2007) proposed the 'environmental heterogeneity hypothesis of invasions' that heterogeneity should both increase the success of invading species and decrease the impact on native species by promoting coexistence mechanisms that are not present otherwise. We use our model to elucidate some of the mechanistic underpinnings for this hypothesis and show how movement behaviour in patchy landscapes affects invasion speeds and coexistence opportunities.

We formulate a two-species competition model in a patchy landscape, consisting of two types of habitat patches. Since the invasion speed for our model cannot be calculated explicitly, we use homogenization as an analytical technique and compare the results with numerical simulations. We investigate how habitat-specific movement rates and habitat preference influence the establishment and rates of spatial spread of an invading species. Comparisons with numerical solutions indicate that the derived speed provides a good approximation even beyond the small-scale heterogeneity limit of the homogenization. We choose population dynamic parameters in such a way that the two species cannot coexist in any isolated patch. When invasion is successful, we observe cases in which the resident is replaced and cases where both species coexist. These outcomes as well as the invasion speeds are found to be strongly dependent on movement behaviour.

\section{The model equations}

Following Shigesada et al. (1986), we divide the infinite, one-dimensional, landscape into periodically alternating patches of two types. Patches of type 1 are of length $l_{1}$ and are located at $x \in \Omega_{1}=\bigcup_{n}\left(n l, n l+l_{1}\right)$, with $n \in \mathbb{Z}$. Analogously, patches of type 2 with length $l_{2}$ are at $x \in \Omega_{2}=\bigcup_{n}\left(n l+l_{1},(n+1) l\right)$. The landscape period is $l=l_{1}+l_{2}$. We denote by $u_{1,2}(x, t)$ the population densities of species 1 and 2, respectively, at position $x \in \mathbb{R}$ and time $t>0$. Adopting the landscape ecology perspective, we choose the movement and growth parameters to be piecewise constant. Thus, the reaction-diffusion system for $x \in \Omega_{j}, j \in\{1,2\}$, reads

$$
\begin{aligned}
& \frac{\partial u_{1}}{\partial t}=d_{1 j} \frac{\partial^{2} u_{1}}{\partial x^{2}}+f_{1 j}\left(u_{1}, u_{2}\right), \\
& \frac{\partial u_{2}}{\partial t}=d_{2 j} \frac{\partial^{2} u_{2}}{\partial x^{2}}+f_{2 j}\left(u_{1}, u_{2}\right)
\end{aligned}
$$

where functions $f_{i j}$ account for births and deaths of population $i=1,2$ in a patch of type $j . d_{1 j}\left(d_{2 j}\right)$ is the diffusion constant of species 1 (species 2 ) in $\Omega_{j}$. In the next step, we define how the equations on each patch are related across patch boundaries.

\section{Interface conditions}

At an interface between patches, individuals may show preference for one or the other patch type. Accordingly, we impose conditions that relate the population densities and fluxes on either side of the interface. Until very recently, the standard assumption was that densities and population flux should be continuous across interfaces (Ludwig et al. 1979; Shigesada et al. 1986; Cruywagen et al. 1996; Lutscher et al. 2006). However, Ovaskainen and Cornell (2003) showed that although flux continuity is a natural condition, indicating that all individuals that leave one patch must enter the next, the densities across interfaces are typically discontinuous. The mathematical and ecological consequences of these discontinuities were discussed by Maciel and Lutscher (2013). The interface conditions can be derived from a random walk model, similar to the derivation of the diffusion equation (Turchin 1998). We briefly outline the main assumptions and procedures used. For a complete derivation, we refer the reader to the original work by Ovaskainen and Cornell (2003) and Maciel and Lutscher (2013). An alternative, heuristic derivation can be found in Maciel and Lutscher (2015).

We consider an uncorrelated, unbiased random walk of an individual of species $i$ on a line. In the interior of patch type 1 (patch type 2), during a time step of length $\Delta t$, the individual moves a distance $\Delta x$ to the left or right with equal probability $p_{i 1} / 2\left(p_{i 2} / 2\right)$. The so-called 'master equation' for this process tracks the probability of an individual's location from one time step to the next (Turchin 1998). 
Taking the 'parabolic limit' of this master equation as $\Delta t, \Delta x \rightarrow 0$, one obtains the diffusion equation with diffusion coefficient (Turchin 1998)

$0<d_{i j}=\lim _{\Delta t, \Delta x \rightarrow 0} \frac{p_{i j}(\Delta x)^{2}}{2 \Delta t}<\infty$.

At an interface point, we assume that individuals can show movement bias. Accordingly, we denote by $\alpha_{i} \in(0,1)$ the probability that an individual of species $i$ located at an interface between two habitat types moves to patch type 1 and by $1-\alpha_{i}$ the probability that it moves to patch type 2 . As before, one can write the master equation and take the parabolic limit. This time, one obtains relations between the population density and flux at either side of the interface. At an interface point $x_{n}=n l+l_{1}(n \in \mathbb{Z})$ with a patch of type 1 to the left and a patch of type 2 on the right, the resulting matching conditions for species $i$ are

$$
\begin{aligned}
& d_{i 1} \frac{\partial u_{i}}{\partial x}\left(x_{n}^{-}, t\right)=d_{i 2} \frac{\partial u_{i}}{\partial x}\left(x_{n}^{+}, t\right) \\
& u_{i}\left(x_{n}^{-}, t\right)=k_{i} u_{i}\left(x_{n}^{+}, t\right), \quad k_{i}=\frac{\alpha_{i}}{1-\alpha_{i}} \frac{d_{i 2}}{d_{i 1}},
\end{aligned}
$$

where $u\left(x_{n}^{ \pm}\right)=\lim _{x \rightarrow x_{n}^{ \pm}} u(x)$ denote the one-sided limits. Equation (9) is the flux conservation while (10) gives the density jump across the interface. We also call the nondimensional parameter $k_{i}$ the 'effective patch preference'. Note that densities are discontinuous even when there is no movement bias (i.e. $\alpha_{i}=0.5$ ) as long as the diffusion coefficients differ between patches. At the interfaces $x_{n}=$ $n l$, where a patch of type 2 is on the left side of the boundary, similar conditions apply. Conditions are then written as (9) and (10) with the signs of the one-sided limits reversed.

\section{Population dynamics}

In patches of type 1 , we choose $f_{i 1}$ as the classical LotkaVolterra system so that the reaction-diffusion system reads

$$
\begin{aligned}
& \frac{\partial u_{1}}{\partial t}=d_{11} \frac{\partial^{2} u_{1}}{\partial x^{2}}+r_{11} u_{1}\left(1-\frac{u_{1}}{K_{11}}-a_{12} \frac{u_{2}}{K_{11}}\right) \text { in } \Omega_{1}, \\
& \frac{\partial u_{2}}{\partial t}=d_{21} \frac{\partial^{2} u_{2}}{\partial x^{2}}+r_{21} u_{2}\left(1-\frac{u_{2}}{K_{21}}-a_{21} \frac{u_{1}}{K_{21}}\right) \text { in } \Omega_{1},
\end{aligned}
$$

where $r_{i j}>0$ and $K_{i j}>0$ are, respectively, the growth rate and carrying capacity of species $i$ in a patch of type $j$, whereas $a_{12}>0$ and $a_{21}>0$ are the inter-specific competition coefficients. In particular, since $r_{i 1}>0$, each species in isolation can persist on a patch of type 1 . These are 'source' patches; we also call them 'strongly favourable' (SF).

For patches of type 2, we consider two distinct scenarios: mildly favourable (MF) and unfavourable (U). Mildly favourable patches are population dynamic sources (i.e. $r_{i 2}>0$ ), but of lesser quality than strongly favourable patches. We choose the same Lotka-Volterra functions for $f_{i 2}$ but with lower carrying capacities. Unfavourable patches are population dynamic sinks. We neglect competition in unfavourable patches and simply write $f_{i 2}=$ $-m_{i 2} u_{i}$, where $m_{i 2}$ is the mortality rate of species $i$ in $\Omega_{2}$.

It is tempting, and even helpful, to write the model (7) and (8) in the condensed form for $x \in \mathbb{R}$

$$
\begin{aligned}
\frac{\partial u_{1}}{\partial t} & =\frac{\partial^{2}}{\partial x^{2}}\left[d_{1}(x) u_{1}\right]+f_{1}\left(x, u_{1}, u_{2}\right), \\
\frac{\partial u_{2}}{\partial t} & =\frac{\partial^{2}}{\partial x^{2}}\left[d_{2}(x) u_{2}\right]+f_{2}\left(x, u_{1}, u_{2}\right),
\end{aligned}
$$

where

$d_{i}(x)=\left\{\begin{array}{ll}d_{i 1} & \text { in } \Omega_{1}, \\ d_{i 2} & \text { in } \Omega_{2},\end{array}\right.$ and $f_{i}\left(x, u_{1}, u_{2}\right)= \begin{cases}f_{i 1}\left(u_{1}, u_{2}\right) & \text { in } \Omega_{1}, \\ f_{i 2}\left(u_{1}, u_{2}\right) & \text { in } \Omega_{2} .\end{cases}$

In the following sections, this form will be assumed from time to time. We would like to point out, however, that these equations are not well defined at the interface points and that the notion of a 'solution' requires the interface conditions (9)-(10). The existence of appropriate solutions was recently proved (Maciel et al. 2018).

\section{Methods}

Ideally, we would consider the linearization of system (13)-(14) at the semi-trivial state where one species is at carrying capacity and the other is absent, and calculate the persistence condition and spread rate of the other species. However, since the single-species steady state is not explicitly available, and even if it were, the spread rate could not be calculated explicitly, we choose a different route. Specifically, we use multi-scale analysis and homogenization. The theory of these techniques is mathematically well developed (Pavliotis and Stuart 2008) and profitably used in many physical and some biological applications (e.g. Othmer 1983), but has-despite recent success (Duncan et al. 2017; Garlick et al. 2011; Garlick et al. 2014; Powell and Zimmermann 2004)—not yet reached its full potential in theoretical ecology.

The underlying idea is that there are two spatial scales: the small scale of landscape heterogeneity and the larger scale of dispersal. The complicated exact equations that consider both scales are then replaced by simpler approximate equations on the larger scale, where the effects on the smaller scale enter as appropriate averages in the parameters for the equation on the larger scale. The usefulness from this technique stems from the fact that the results derived from the equations on the large scale, while valid formally only in the limit when the scale difference is 
large, typically provide highly accurate results even when the scales are almost comparable.

The classical theory of homogenization assumes smoothly varying conditions, yet the patchy landscape that we model contains discontinuities and interface conditions. Our previous approach for single equation (Maciel and Lutscher 2015) does not carry over to the system of equations here; however, recent progress by Yurk and Cobbold (2018) does and was already used for stage-structured populations (Alqawasmeh 2017). We outline the procedure here and refer to the literature for details.

We assume that the spatial period $l$ in (13)-(14) is small compared to the scales of observation and write $\epsilon=l \ll 1$. We identify two distinct spatial scales, a large scale $x$ and a small scale $y=x / \epsilon$ (Othmer 1983; Garlick et al. 2011). We furthermore assume that diffusion and growth depend only on the small scale, i.e. $d_{i}=d_{i}(y)$ and $f_{i}=f_{i}\left(y, u_{i}, u_{j}\right)$. Population densities depend on both, small and large, scales so that we formally write $u_{i}=u_{i}(x, y, t)$. We expand solutions in power series of $\epsilon$, i.e.

$u_{i}(x, y, t)=u_{i}^{(0)}(x, y, t)+\epsilon u_{i}^{(1)}(x, y, t)+\epsilon^{2} u_{i}^{(2)}(x, y, t)+\ldots$

Plugging (15) into (13)-(14), we obtain equations for the different orders in $\epsilon$ that can be solved iteratively, yet the procedure is very tedious and quite delicate. Yurk and Cobbold (2018) and Alqawasmeh (2017) show that the leading term solution is given by

$u_{i}^{(0)}(x, y, t)=\frac{g_{i}(x, t)}{h_{i}(y)}$,

where $g_{i}$ solves the homogeneous reaction-diffusion equation:

$\frac{\partial g_{i}}{\partial t}=\hat{l}_{i}^{2}\left\langle d_{i}\right\rangle_{H} \frac{\partial^{2} g_{i}}{\partial x^{2}}+\left\langle f_{i}\right\rangle_{A}$,

and $h_{i}$ is the step function

$h_{i}(y)= \begin{cases}1 & \text { if } y \text { in } \bar{\Omega}_{1} \\ k_{i} & \text { if } y \text { in } \bar{\Omega}_{2},\end{cases}$

where $\bar{\Omega}_{i}=\Omega_{i} / \epsilon$. The averaged parameters in the equations for $g_{i}$ are the scaled period

$\hat{l}_{i}=\frac{l_{1}+l_{2}}{l_{1}+l_{2} / k_{i}}$,

the effective diffusion (the harmonic mean)

$\left\langle d_{i}\right\rangle_{H}=\left(\frac{l_{1}+l_{2} / k_{i}}{\frac{l_{1}}{d_{i 1}}+\frac{l_{2} / k_{i}}{d_{i 2} / k_{i}^{2}}}\right)$, and the arithmetic mean of the growth functions

$\left\langle f_{i}\right\rangle_{A}=\left[\frac{l_{1} f_{i 1}\left(g_{1}, g_{2}\right)+l_{2} f_{i 2}\left(g_{1} / k_{1}, g_{2} / k_{2}\right)}{l_{1}+l_{2} / k_{i}}\right]$.

Powell and Zimmermann (2004) gave a nice intuitive explanation for the appearance of the different averages above in terms of residence times, but in the absence of interface conditions (i.e. when $k=1$ ). Residence time in a patch is proportional to patch size $\left(l_{i}\right)$, and the growth rate $\left(f_{i}\right)$ is measured per unit time. Therefore, the average growth on a patch is the arithmetic mean, $\left(l_{1} f_{1}+\right.$ $\left.l_{2} f_{2}\right) / l$. Residence time is also inversely proportional to the diffusion constant. Hence, the mean residence time is proportional to $\left(l_{1} / d_{1}+l_{2} / d_{2}\right) / l$. The inverse of this expression is the diffusion coefficient on the larger scale, the harmonic mean of the diffusion coefficients on the small scale.

With three different patch types (SF, MF, U) and four possible outcomes of competition on source patches, our model allows for too many combinations to exhaustively classify the dynamic behaviour in all of them. Instead, we focus on the "environmental heterogeneity hypothesis of invasions' (Melbourne et al. 2007) and carefully choose our setup to investigate the effects of movement rates $\left(d_{i j}\right)$ and patch-type preference $\left(\alpha_{i}\right)$ on whether and how fast a competing species can invade an existing resident. By exchanging the role of resident and invader, we examine whether successful invasion leads to replacement or coexistence. We consider two scenarios where coexistence is impossible, locally or regionally, in the absence of movement.

\section{Results}

In what follows, we will study the equations for $g_{i}$ in (17) as indicated above: we linearize at the semi-trivial state and calculate persistence conditions and spread rates from that, using the theory from Lewis et al. (2002). We note that the homogenization approach in (17) is valid for a large class of nonlinear growth functions. Our particular choice of LotkaVolterra (polynomial) interactions, however, is particularly suitable for the averaging process because the arithmetic average of the polynomials is again a polynomial of the same degree, so that we remain within the class of equations studied by Lewis et al. (2002).

\section{Both patches are sources}

In this scenario, the landscape consists of strongly and mildly favourable patches ('sources'). Each species can sustain its population in the absence of the other on each patch (type). The dynamics are given by (11)-(12) with 
competition coefficients being equal on both patch types but carrying capacity being lower on MF than on SF patches.

\section{Setup and simplifications}

We reduce the number of parameters in our model by non-dimensionalization, and we make a few simplifying assumptions. In terms of the non-dimensional quantities

$$
\begin{aligned}
& U_{1}=\frac{u_{1}}{K_{11}}, \quad U_{2}=\frac{u_{2}}{K_{21}}, \quad R_{i j}=\frac{r_{i j}}{r_{11}}, \quad D_{i j}=\frac{d_{i j}}{d_{11}}, \\
& T=r_{11} t, \quad X=x \sqrt{\frac{r_{11}}{d_{11}}}, \quad L_{i}=l_{i} \sqrt{\frac{r_{11}}{d_{11}}}
\end{aligned}
$$

the scaled equations on SF patches read

$$
\begin{aligned}
& \frac{\partial U_{1}}{\partial T}=\frac{\partial^{2} U_{1}}{\partial X^{2}}+U_{1}\left(1-U_{1}-A_{12} U_{2}\right) \quad \text { in } \Omega_{1} \\
& \frac{\partial U_{2}}{\partial T}=D_{21} \frac{\partial^{2} U_{2}}{\partial X^{2}}+R_{21} U_{2}\left(1-U_{2}-A_{21} U_{1}\right) \quad \text { in } \Omega_{1}
\end{aligned}
$$

and on MF patches are given by

$$
\begin{array}{ll}
\frac{\partial U_{1}}{\partial T}=D_{12} \frac{\partial^{2} U_{1}}{\partial X^{2}}+R_{12} U_{1}\left(1-\frac{U_{1}}{K_{1}}-A_{12} \frac{U_{2}}{K_{1}}\right) & \text { in } \Omega_{2} \\
\frac{\partial U_{2}}{\partial T}=D_{22} \frac{\partial^{2} U_{2}}{\partial X^{2}}+R_{22} U_{2}\left(1-\frac{U_{2}}{K_{2}}-A_{21} \frac{U_{1}}{K_{2}}\right) & \text { in } \Omega_{2} .
\end{array}
$$

The remaining non-dimensional parameters are $A_{12}=$ $a_{12} \frac{K_{21}}{K_{11}}, A_{21}=a_{21} \frac{K_{11}}{K_{21}}, K_{1}=\frac{K_{12}}{K_{11}}$ and $K_{2}=\frac{K_{22}}{K_{21}}$. Since the carrying capacities on MF patches are lower, we have $K_{1}, K_{2}<1$. To write interface conditions (9)-(10) in nondimensional quantities, we simply need to replace $d_{i j}$ with $D_{i j}$ (setting $D_{11}=1$ ).

We now apply the homogenization procedure (described earlier), with a series expansion solution in $\epsilon=L=L_{1}+$ $L_{2}$, to each of the two densities of the competition system (21)-(24). The lowest-order term of the solution according to (16) is given by

$U_{i}^{(0)}(X, Y, T)=\frac{\bar{g}_{i}(X, T)}{\bar{h}_{i}(Y)}$,

where $Y=X / \epsilon$ is the small-scale variable. The timedependent function $\bar{g}_{i}(X, T)$ depends only on the largescale variable and is obtained from (17) written in the dimensionless variables. It turns out that under the rescaling

$$
\begin{aligned}
& \hat{g}_{1}=\left(\frac{L_{1}+R_{12} L_{2} /\left(K_{1} k_{1}^{2}\right)}{L_{1}+R_{12} L_{2} / k_{1}}\right) \bar{g}_{1} \quad \text { and } \\
& \hat{g}_{2}=\left(\frac{R_{21} L_{1}+R_{22} L_{2} /\left(K_{2} k_{2}^{2}\right)}{R_{21} L_{1}+R_{22} L_{2} / k_{2}}\right) \bar{g}_{2},
\end{aligned}
$$

the homogenized equations have the same form as (2)-(3), namely

$$
\begin{aligned}
& \frac{\partial \hat{g}_{1}}{\partial T}=D_{1} \frac{\partial^{2} \hat{g}_{1}}{\partial X^{2}}+R_{1} \hat{g}_{1}\left(1-\hat{g}_{1}-A_{1} \hat{g}_{2}\right), \\
& \frac{\partial \hat{g}_{2}}{\partial T}=D_{2} \frac{\partial^{2} \hat{g}_{2}}{\partial X^{2}}+R_{2} \hat{g}_{2}\left(1-\hat{g}_{2}-A_{2} \hat{g}_{1}\right) .
\end{aligned}
$$

Here, $D_{1}=\hat{L}_{1}^{2}\left\langle D_{1}\right\rangle_{H}$ and $D_{2}=\hat{L}_{2}^{2}\left\langle D_{2}\right\rangle_{H}$ are the homogenized diffusion coefficients, with $\hat{L}_{i}$ and $\left\langle D_{i}\right\rangle_{H}$ given as in (18) and (19). The growth rates and competition coefficients in the homogenized model are given by

$$
\begin{gathered}
R_{1}=\left(\frac{L_{1}+R_{12} L_{2} / k_{1}}{L_{1}+L_{2} / k_{1}}\right), \quad R_{2}=\left(\frac{R_{21} L_{1}+R_{22} L_{2} / k_{2}}{L_{1}+L_{2} / k_{2}}\right) \\
A_{1}=A_{12}\left(\frac{L_{1}+R_{12} L_{2} /\left(K_{1} k_{1} k_{2}\right)}{R_{21} L_{1}+R_{22} L_{2} /\left(K_{2} k_{2}^{2}\right)}\right)\left(\frac{R_{21} L_{1}+R_{22} L_{2} / k_{2}}{L_{1}+R_{12} L_{2} / k_{1}}\right) \\
\text { and } A_{2}=A_{21}\left(\frac{R_{21} L_{1}+R_{22} L_{2} /\left(K_{2} k_{1} k_{2}\right)}{L_{1}+R_{12} L_{2} /\left(K_{1} k_{1}^{2}\right)}\right) \\
\times\left(\frac{L_{1}+R_{12} L_{2} / k_{1}}{R_{21} L_{1}+R_{22} L_{2} / k_{2}}\right)
\end{gathered}
$$

We can apply the theory in Lewis et al. (2002) to (27)-(28). In particular, species 1 can invade the resident-only (species 2) steady state if $A_{1}<1$, and under the conditions stated in the introduction, the spread rate of species 1 invading resident species 2 at equilibrium is

$C=2 \sqrt{D_{1} R_{1}\left(1-A_{1}\right)}$.

To illustrate our results, we make the additional simplifying assumption $K_{1}=K_{2}(<1)$ so that the outcome of local competition is determined by the coefficients $A_{i j}$ only. Resident species 2 wins the competition in both patch types if $A_{21}<1<A_{12}$; the invading species 1 wins in both patches if the inequalities are reversed, i.e. $A_{12}<1<A_{21}$. We begin exploring how invasion conditions and spread rates depend on the diffusion coefficients of the two species in each patch.

\section{The effect of diffusion rates}

For the scenario when the resident is the stronger competitor, the stability results are illustrated in Fig. 1a. The horizontal axis shows the ratio of movement rates in bad versus good patches for the resident, the vertical axis for the invader. The light grey region indicates the set $A_{1}>1$. Here, the resident semi-trivial state is stable, the invasion fails, and the resident wins. In the white regions, the inequality is reversed and species 1 can invade. Since, in addition, we have $A_{2}<1$ in the entire region, the semi-trivial invader state is always unstable. Hence, the white regions indicate coexistence of resident and invader. We see that species 1 has no chance to invade if the resident has roughly equal 


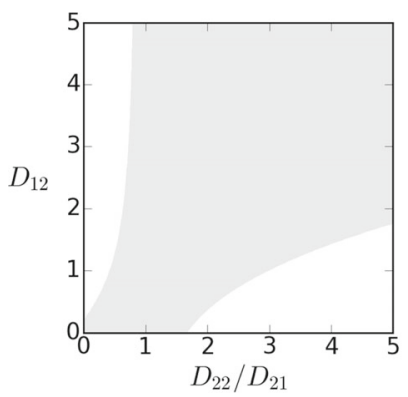

(a)

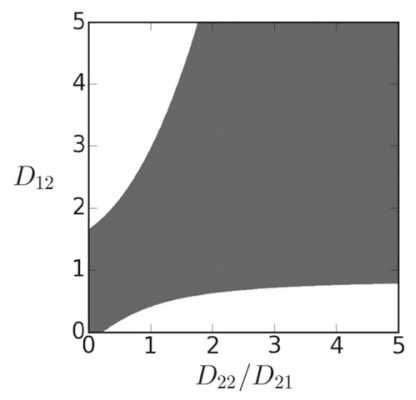

(b)
Fig. 1 Competition outcomes as a function of the resident and invader diffusivity ratios, $D_{22} / D_{21}$ and $D_{12}$, respectively. In light (dark) grey regions, species 2 (1) wins; in white regions, coexistence is possible. In $\mathbf{a}$, the resident is the stronger competitor in both patch types with $A_{21}=0.8<1<1.2=A_{12}$. In $\mathbf{b}$, the invader is the stronger competitor in both patch types as we set $A_{12}=0.8<1<1.2=$ $A_{21}$. At interfaces, individuals choose each habitat type with equal probability, i.e. $\alpha_{1}=\alpha_{2}=0.5$. The remaining (default) parameters are $L_{1}=L_{2}=1, R_{12}=R_{21}=R_{22}=1$ and $K_{1}=K_{2}=0.8$

mobility in both patch types. If, however, the resident moves much faster in one of the two patch types, then the species 1 can invade when it moves much slower in that patch type. Since movement rates are inversely correlated with residence times, we can also say that if the residence time of the resident in one patch type is much higher than in the other, then the invader can be successful by choosing its residence times in the opposite way. Similar considerations apply in the other scenario when the invader species 1 is the better competitor in both patch types. Figure $1 \mathrm{~b}$ indicates this scenario, with the dark grey region representing the exclusion of the resident and the white region representing coexistence as above.

We now illustrate how the spread rate of the invader depends on the diffusion rate of the resident in bad patches, $D_{22}$. While the competition outcome depends on movement rates only through the ratios $D_{22} / D_{21}$ and $D_{12}$, as can be seen from (30) and (31), the speed does not. We plot the spread speed from the homogenization formula in (32) and compare it with the results of numerical simulations of the original system, obtained by a backwards Euler, finite difference scheme (Strikwerda 2004). In all cases, the homogenization approximation (solid lines) and the numerical speed (black dots) agree very well, even though the period of the landscape is not particularly small compared to the diffusivities.

We begin again with the scenario where the resident is the better competitor in both patch types, and we fix the invader diffusion constant to $D_{12}=1$, i.e. the invader moves at the same rate in both patch types. Its spread rate first decreases with $D_{22}$ until it hits zero where the invasion is not successful. For large enough values of $D_{22}$ the spread rate increases again; see Fig. 2a. When the invader is the better competitor in both patch types, the speed is positive across the range of $D_{22}$, but has a local minimum at intermediate values; see Fig. 2b. Hence, in both cases, the slowest speeds occur when the resident moves similarly in the two patch types; faster speeds are possible when the resident uses different movement rates.

\section{The effect of habitat preference}

So far, we only considered movement rates of the two species. Now we illustrate the influence of habitat preference. Figure 3 illustrates the regions of coexistence (white), species 2 dominance (light grey) and species 1 dominance (dark grey) as before. Figure $3 \mathrm{a}$ is for the scenario where the resident is the stronger species in both patch types. We see again that the two species are more likely to coexist if their habitat preferences are more dissimilar. Figure $3 \mathrm{~b}$ shows the case where the invader is the stronger competitor in both patch types. The corresponding spread rates (for $\alpha_{1}=0.5$ ) are illustrated in Fig. 4a, b, respectively. We see a similar pattern as before: the invasion is fastest when the resident patch preference is very high or very low, and it spreads more slowly (or not at all) when the resident patch preference is intermediate.
Fig. 2 Spread speed of species 1 (with $D_{12}=1$ ) as a function of species 2 diffusivity in unfavourable patches, $D_{22}$. In a, species 2 is the stronger competitor in both patch types $\left(A_{21}=0.8<1<1.2=A_{12}\right)$. In $\mathbf{b}$, species 1 outcompetes 2 in both patch types

$\left(A_{12}=0.8<1<1.2=A_{21}\right)$.

We set $D_{21}=1$ and all other parameters are the default values from Fig. 1

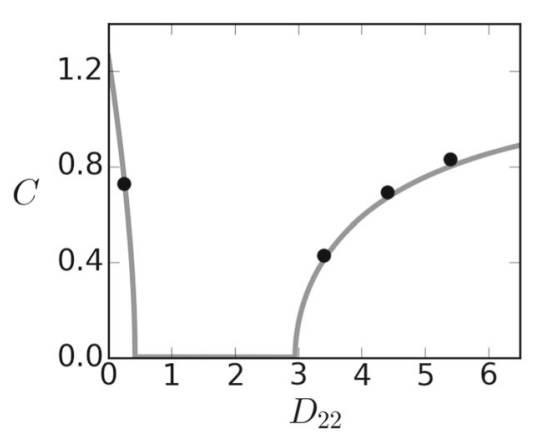

(a)

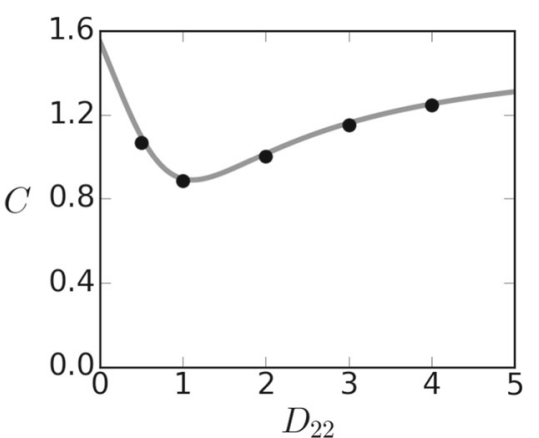

(b) 


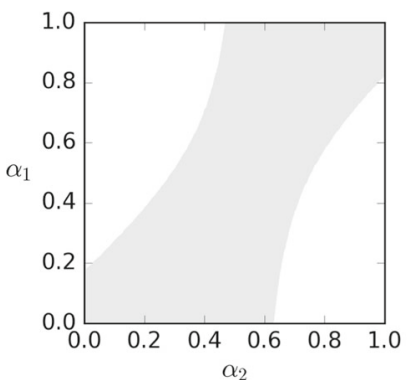

(a)

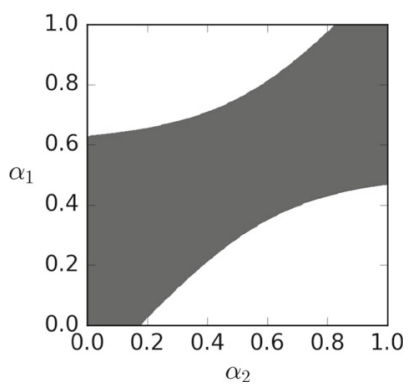

(b)
Fig. 3 Competition outcomes as a function of habitat preference of resident and invader species. White regions correspond to a coexistence state. In light (dark) grey regions, species 2 (1) wins. In $\mathbf{a}$, the resident species wins local competition in both patches $\left(A_{21}=0.8<1<1.2=A_{12}\right)$. In $\mathbf{b}$, the invader wins both local competitions $\left(A_{12}=0.8<1<1.2=A_{21}\right)$. Movement parameters are $D_{12}=D_{21}=D_{22}=1$; all other parameters are set at the default values from Fig. 1

\section{The effect of carrying capacities}

Next, we look at how the scenarios of coexistence and exclusion depend on the difference in habitat quality between SF and MF patches. We choose $K_{1}=K_{2}=K$ as our control parameter. When $K$ is near one, MF are almost as good as SF patches. When $K$ is near zero, MF patches are much worse than SF patches. Figure $5 \mathrm{a}, \mathrm{b}$ shows how the resulting competitive outcome depends on the resident species' movement rates. When the resident species is the stronger competitor (Fig. 5a), the invader can replace it if $K$ is low and the resident moves slowly in MF patches (low $D_{22} / D_{21}$ ). For high $K$, the (stronger) resident cannot be replaced but the two species will coexist for small or large $D_{22} / D_{21}$. When the invader is the stronger competitor (Fig. 5b), it will always exclude the resident for high $K$. The invader is eliminated at small $K$ and high $D_{22} / D_{21}$. Coexistence arises at intermediate $K$ and large $D_{22} / D_{21}$.

\section{Similar competitors}

In previous sections, we had chosen the competition coefficients 'far away' from unity, so that the species had a relatively strong competitive (dis-)advantage. In that case, we saw that movement behaviour in a heterogeneous environment could lead to coexistence of the two species even though one species dominated the local competition in both patch types. Here we choose the competition coefficients closer to unity so that the competitive advantage is weak. We shall see that in addition to the scenarios presented before, bistability can also arise.

The setup in Fig. 6 is similar to the one in Fig. $1 b$. The resident is the weaker competitor in both patch types, but this time, the competitive disadvantage is small. In comparing the two figures, we see that the region in which the invader excludes the resident is smaller and a new region emerges where the inferior resident excludes the invader. The light grey region where the resident wins may be separated from the dark grey region where the invader wins by the white region of coexistence (Fig. 6a), or it may overlap, leading to the diagonally striped region of bistability (Fig. 6b). In these figures, neither species shows habitat preference, i.e. $\alpha_{i}=0.5$, but the same qualitative behaviour can be observed when the diffusion rates are constant and equal in both patch types, and the habitat preferences vary (similar to Figs. 3, plots not shown).

We conclude that if the competitive advantage is relatively large (Fig. 1), the stronger competitor has nothing to fear: it cannot be replaced (irrespective of movement strategy); it just may not be able to eliminate the weaker competitor (depending on movement strategy). When the competitive advantage is relatively small, though (Fig. 6), the stronger competitor faces the risk of elimination due solely to the chosen movement strategies.

We use again the formula in (32) to calculate the spread rate of species 1 into the resident species 2 (and vice versa). We fix the diffusion coefficient of species 1 in bad patches to $D_{12}=5$. The results are depicted in Fig. 7. Similarly to
Fig. 4 Invader spread rates as a function of resident habitat preference $\alpha_{2}$. Competitive scenarios correspond to those in Fig. 3,

i.e. $A_{21}=0.8<1<1.2=A_{12}$ in $\mathbf{a}$ and

$A_{12}=0.8<1<1.2=A_{21}$ in

b. In both cases, we have

$\alpha_{1}=0.5$

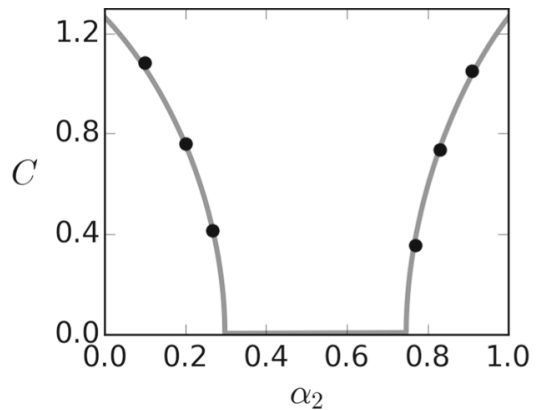

(a)

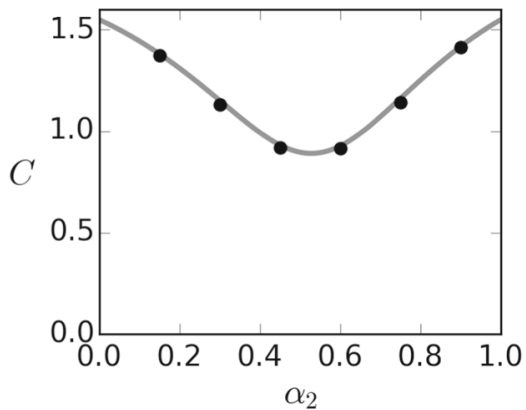

(b) 
Fig. 5 Competition outcomes as a function of resident diffusion rates and carrying capacities in lower quality patches. In light (dark) grey regions, species 2 (1) wins; in white regions, coexistence is possible. Competition coefficients are as in previous figures,

i.e. $A_{21}=0.8<1<1.2=A_{12}$ in $\mathbf{a}$ and

$A_{12}=0.8<1<1.2=A_{21}$ in

b. We have $D_{12}=1$,

$\alpha_{1}=\alpha_{2}=0.5$ and other

parameters at default values

Fig. 2, the speed of species 1 is minimal for intermediate values of $D_{22}$. In contrast to Fig. $2 \mathrm{~b}$, the speed even reaches zero so that invasion is impossible even though species 1 is locally the better competitor in both patch types. When $D_{22}$ is small, species 1 spreads quickly since it uses mostly the SF habitat type whereas species 2 uses mostly the MF habitat type. When $D_{22}$ is large, species 1 also spreads quickly since it uses the habitat in a similar way to species 2 and it is the superior competitor. For intermediate values of $D_{22}$, species 1 loses its local competitive advantage. Since it moves quickly in MF habitat, its density there is so low that species 2 can persist there and even outcompete species 1 globally.

The spread rate of species 2 (dashed lines in Fig. 7) is a hump-shaped function of $D_{22}$. Clearly, when $D_{22}=0$, the

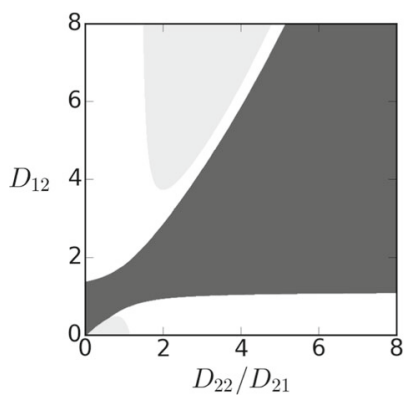

(a)

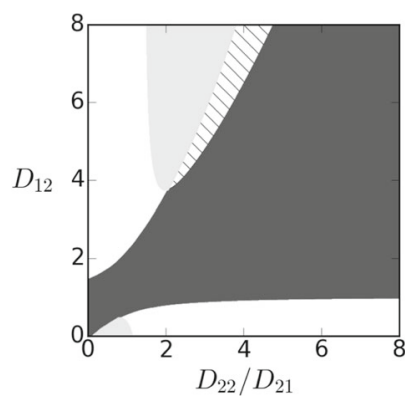

(b)
Fig. 6 Competition outcomes as a function of the resident and invader diffusivity ratios, $D_{22} / D_{21}$ and $D_{12}$, respectively. In light (dark) grey regions, species 2 (1) wins; in white regions, coexistence is possible; in the diagonally striped region, we have founder control. Please note that, because of the scale, the behaviour in the bottom left corner is difficult to see. In a, the light and dark grey regions are separated by a very narrow white region of coexistence; in $\mathbf{b}$, the light and dark grey regions overlap and create a region of bistability. In both cases, the invader is the stronger local competitor in both patch types with $A_{12}=0.95<1<1.05=A_{21}$ in a and $A_{12}=0.95<1<1.1=A_{21}$ in $\mathbf{b}$. At interfaces, individuals choose each habitat type with equal probability, i.e. $\alpha_{1}=\alpha_{2}=0.5$. The remaining (default) parameters are $L_{1}=L_{2}=1, R_{12}=R_{21}=R_{22}=1$ and $K_{1}=K_{2}=0.8$ species cannot spread. When $D_{22}$ is small, species 2 spends most of the time in MF habitat and uses the resources there so well that it can spread into species 1 and even outcompete it. As $D_{22}$ increases further, species 2 spends more time in SF habitat, just as species 1, and it loses the local competition there. The spread rate decreases and eventually becomes zero. If the species 2 spread rate becomes zero before the species 1 rate becomes positive (Fig. 7b), we have a bistable situation; otherwise, we have a coexistence region (Fig. 7a).

Please note that we indicate by the cross-hatched region the parameter range for which the sufficient conditions for linear determinacy in (6) are not satisfied. Our simulations show that the linearized formula still predicts the numerically simulated spread rate very well.

The various outcomes that we found depend in complex ways on species behaviour and environmental attributes. This complexity can be expected in models with many parameters. Our goal, however, is to elucidate the role of movement patterns in the competitive outcome. The key to this understanding is the observation that a species' occupancy in a type 1 patch increases if its patch preference $\alpha$ increases and/or its relative movement rate in patch type 1 decreases. In other words, occupancy times in a SF patch is high (low) if the 'effective patch preference' $\left(k_{i}\right)$ is high (low). In Table 1, we summarize the conditions for the different competitive outcomes based on patch occupancy. Note that on this level of abstraction, the conditions for founder control are the same as for competitive reversal; the detailed differences between the two depend on parameter values.

\section{Unfavourable patches are sinks}

We now consider the scenario of a source-sink landscape: patches of type 1 are strongly favourable (SF) and patches of type 2 are unfavourable (U). In SF patches, we have LotkaVolterra competition and the (rescaled) dynamics are as in 
Fig. 7 Spread speed of species 1 (solid) and species 2 (dashed) into its respective competitor as a function of species 2 diffusivity in bad patches, $D_{22}$. Species 1 locally outcompetes 2 in both patch types with a $A_{12}=0.95<1<1.05=A_{21}$ and $\mathbf{b}$

$A_{12}=0.95<1<1.1=A_{21}$. Parameters are $D_{12}=5$,

$D_{21}=1$ and all other parameters are the default values from Fig. 1

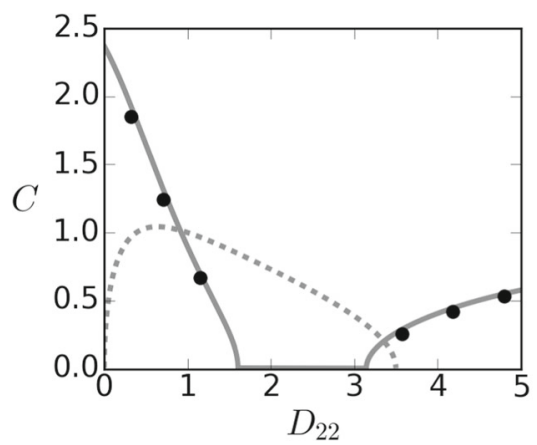

(a)

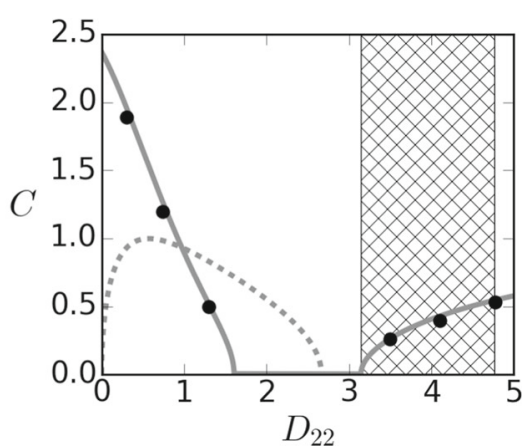

(b)
(21) and (22). Due to the presence of U patches, persistence of each species in the absence of the other is conditional on the relative size of $\mathrm{SF}$ and $\mathrm{U}$ patches, the respective growth rates and movement behaviour. We studied these conditions in detail in Maciel and Lutscher (2013). Here, since we focus on competition, we shall always assume that each species can persist in the absence of the other.

\section{Setup and simplification}

As before, we non-dimensionalize the equations to reduce the number of parameters. Non-dimensional equations in $\mathrm{U}$ patches are given by

$\frac{\partial U_{1}}{\partial T}=D_{12} \frac{\partial^{2} U_{1}}{\partial X^{2}}-M_{12} U_{1} \quad$ in $\Omega_{2}$,

$\frac{\partial U_{2}}{\partial T}=D_{22} \frac{\partial^{2} U_{2}}{\partial X^{2}}-M_{22} U_{2}$ in $\Omega_{2}$,

where $M_{12}>0$ and $M_{22}>0$ are the rescaled mortality rates of species 1 and 2, respectively.

Applying the homogenization technique as in the previous scenario, we obtain solutions at leading order in the form of (25). The persistence conditions for each species in isolation in the homogenized equations are $L_{1}-M_{12} L_{2} / k_{1}>0$ and $R_{21} L_{1}-M_{22} L_{2} / k_{2}>0$. They both express that growth on SF patches has to be stronger than loss on $U$ patches.
As mentioned above, we shall henceforth assume that these conditions are satisfied. Instead of the scaling in (26), we now introduce the scaled variables

$\hat{g}_{1}=\frac{L_{1}}{L_{1}-M_{12} L_{2} / k_{1}} \bar{g}_{1} \quad$ and $\quad \hat{g}_{2}=\frac{R_{21} L_{1}}{R_{21} L_{1}-M_{22} L_{2} / k_{2}} \bar{g}_{2}$.

By the persistence conditions, these scalings are positive. We then arrive at homogenized equations for $\hat{g}_{1}$ and $\hat{g}_{2}$, analogous to (27) and (28), namely

$$
\begin{aligned}
& \frac{\partial \hat{g}_{1}}{\partial T}=D_{1} \frac{\partial^{2} \hat{g}_{1}}{\partial X^{2}}+R_{1} \hat{g}_{1}\left(1-\hat{g}_{1}-A_{1} \hat{g}_{2}\right) \\
& \frac{\partial \hat{g}_{2}}{\partial T}=D_{2} \frac{\partial^{2} \hat{g}_{2}}{\partial X^{2}}+R_{2} \hat{g}_{2}\left(1-\hat{g}_{2}-A_{2} \hat{g}_{1}\right)
\end{aligned}
$$

with $D_{1}=\hat{L}_{1}^{2}\left\langle D_{1}\right\rangle_{H}$ and $D_{2}=\hat{L}_{2}^{2}\left\langle D_{2}\right\rangle_{H}$ as before. The homogenized growth rates and competition coefficients are now given by

$$
\begin{aligned}
& R_{1}=\left(\frac{L_{1}-M_{12} L_{2} / k_{1}}{L_{1}+L_{2} / k_{1}}\right) \\
& R_{2}=\left(\frac{R_{21} L_{1}-M_{22} L_{2} / k_{2}}{L_{1}+L_{2} / k_{2}}\right) \\
& A_{1}=\frac{A_{12}}{R_{21}}\left(\frac{R_{21} L_{1}-M_{22} L_{2} / k_{2}}{L_{1}-M_{12} L_{2} / k_{1}}\right) \\
& A_{2}=R_{21} A_{21}\left(\frac{L_{1}-M_{12} L_{2} / k_{1}}{R_{21} L_{1}-M_{22} L_{2} / k_{2}}\right) .
\end{aligned}
$$

Table 1 Summary of competitive outcomes when both patches are sources. Please refer to text for more details

Outcome Conditions

Stronger competitor wins

Weaker competitor wins

Coexistence

Founder control
- SF and MF habitats are similar; effective patch preference of the two species are similar (Figs. 1, 3, and 6). - SF and MF habitats differ substantially; effective patch preference of the weaker competitor is not much higher than the stronger competitor (Fig. 5).

- SF and MF habitats differ substantially; effective patch preference of the weaker competitor is substantially higher than the stronger competitor (Fig. 5).

- Competitive advantage is small; competitors prefer same patch type $\left(k_{1,2}>1\right.$ or $\left.k_{1,2}<1\right)$; inferior competitor occupies both patches more equally (Fig. 6).

- SF and MF habitats are similar; effective patch preference between species differ (Figs. 1, 3, and 6).

- Competitive advantage is small; competitors prefer same patch type $\left(k_{1,2}>1\right.$ or $\left.k_{1,2}<1\right)$; inferior competitor occupies both patches more equally (Fig. 6). 
As above, these expressions are positive because of the condition that each species can persist in isolation.

\section{The effect of diffusion rates}

We proceed with the analysis by focusing on the diffusion rates, analogous to the SF/MF scenario. The solid black rectangles in Fig. 8 indicate that neither species can persist (in isolation) when their movement rates in $\mathrm{U}$ habitat are too low. If the invader has a high enough diffusion rate in $\mathrm{U}$ patches, it will persist and spread in isolation, as indicated by the dotted rectangle on the left of Fig. 8. The dotted rectangle on the lower part of the figure indicates the opposite scenario that $D_{22} / D_{21}$ is high enough for the resident to persist, but $D_{12}$ is too small for the invader to persist alone. As the invader's movement rate in U patches increases, it could persist on its own, but is outcompeted by the resident (light grey region), even though the resident is locally the weaker competitor for the parameter values chosen in Fig. 8. As $D_{12}$ increases even more, the invader will be able to persist and spread, and for high enough values of $D_{12}$ eventually outcompete the resident (dark grey region). The transition between the two scenarios can occur via a coexistence region of mutual invasion (white region in Fig. 8a) or, for slightly different parameter values, via a region of founder control (diagonally striped region in Fig. 8b). Which situation occurs depends on the reverse question of when species 2 can invade species 1 .

The different scenarios are reflected in the plot of the spread rates in Fig. 9. As in the SF/MF scenario before, the speed depends on the diffusion rates individually while competitive outcome depends only on the ratio $D_{22} / D_{21}$. When $D_{22}$ is too small for species 2 to persist,

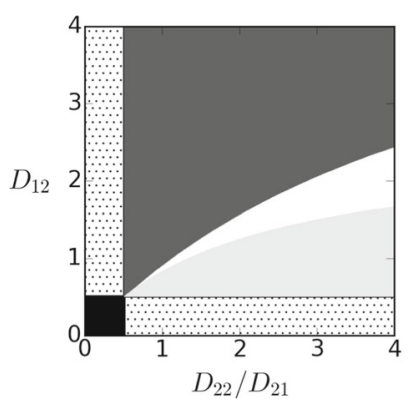

(a)

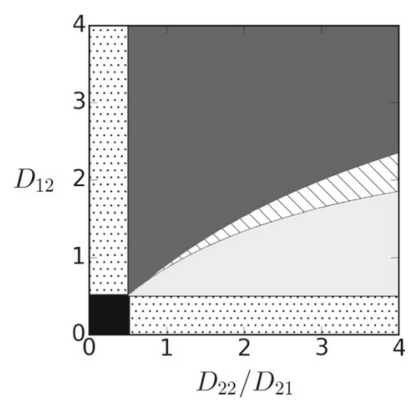

(b)
Fig. 8 Outcome of competition in a source-sink landscape, depending on diffusion ratios $D_{22} / D_{21}$ and $D_{12}$. In light (dark) grey regions, species 2 (1) wins; in the white region, both coexist; the diagonally striped region indicates bistability. The dotted and black areas correspond to one or both species being unable to persist in the absence of the competitor. In both cases, parameters are chosen such that species 1 is the stronger local competitor. Parameter values are a $A_{12}=0.8<1<A_{21}=1.1$ and $\mathbf{b} A_{12}=0.9<1<A_{21}=1.2$. In both cases, we have $M_{i j}=0.5$. Other parameters have the default values from previous figures

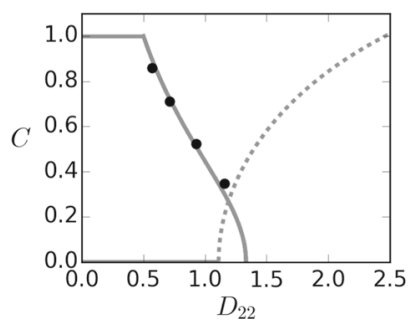

(a)

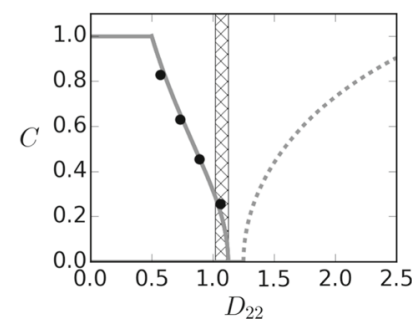

(b)
Fig. 9 The spread rate of species 1 (solid) and species 2 (dashed) as a function of the diffusion constant $D_{22}$, with $D_{21}=1$ and $D_{12}=1$ fixed. a Both species have positive spread rates at intermediate values, so that there is coexistence based on mutual invasion. b The region where both spread rates are zero indicates bistability. The spread rate of species 1 is constant for small values of $D_{22}$ since species 2 is absent from the environment at these values. In the cross-hatched region, the sufficient conditions for linear determinacy in (6) are not satisfied. The remaining parameters are $\mathbf{a} A_{12}=0.8<1<A_{21}=1.1$ and $\mathbf{b}$ $A_{12}=0.9<1<A_{21}=1.2$ and other defaults as before

then the spread rate of species 1 is independent of the motility of species 2 (solid curve). When motility is large enough for the species to persist, then the invader's spread rate decreases as the resident uses some of the resources. Eventually, the invader's speed goes to zero when the resident motility is high. At that point, the resident outcompetes the competitively superior invader. Switching the roles of resident and invader, we obtain the spread rate for species 2 as the dashed curve in Fig. 9. Here, we can clearly distinguish species coexistence from bistability. Coexistence occurs when both species have a positive spread rate for some motility parameters (Fig. 9a), yet bistability arises when the two speeds are never both positive (Fig. 9b). The cross-hatched region in Fig. 9b indicates the parameter range where conditions for linear determinacy in (6) are not satisfied. Yet, simulations show again a very good agreement with the approximate speed.

\section{The effect of habitat preference}

The reasoning about the effects of the patch preference parameters $\alpha_{i}$ is very similar to the preceding case. We only illustrate the coexistence scenario in Fig. 10. When a species has low preference for the good patches, it cannot persist. This region is indicated by the black rectangle (neither can persist) and the dotted regions (only one can persist). If the preference for good patches increases, a species can persist on its own, and it may coexist or outcompete its competitor, depending on parameter values. Coexistence happens when both species have a positive spread rate in the presence of the other species. When the preference for good patches becomes very high, the speed of spread slows down because individuals do not leave good patches any more. In summary, the resident can achieve a competitive 
Fig. 10 Competitive outcome (left plot) and spread rates (right plot) as a function of habitat preference parameters $\alpha_{i}$. Colour patterns are as in the previous two figures. Parameters are $A_{12}=0.8<1<A_{21}=1.1$ for $\mathbf{a}$ and $\mathbf{b}$. We set $\alpha_{1}=0.5$ in $\mathbf{b}$ and the diffusion coefficients are $D_{12}=D_{21}=D_{22}=1$ in both panels

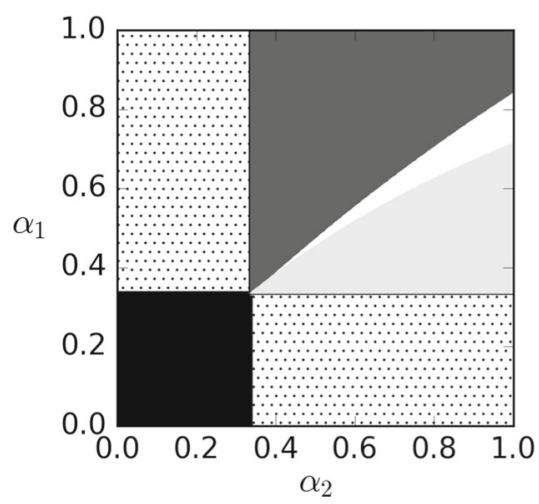

(a)

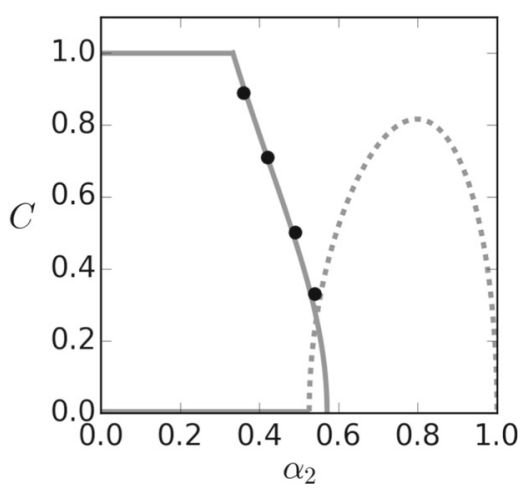

(b) advantage by high diffusion rates in bad patches and/or high preference for good patches. In other words, the outcome of competition is decided again, in large parts, by the effective patch preferences $k_{i}$, defined in (10).

We can explicitly calculate the outcome of competition for two competing species that differ only in their movement behaviour as follows. We make the simplifying assumption that the two species are identical in growth rate $\left(R_{i j}=1\right)$ and mortality $\left(M_{i j}=1\right)$ and that the two patch types are of equal length. Then, the homogenized competition coefficients in (39)-(40) can be written in terms of the effective patch preferences as

$A_{1}=A_{12} \frac{1-1 / k_{2}}{1-1 / k_{1}}, \quad A_{2}=A_{21} \frac{1-1 / k_{1}}{1-1 / k_{2}}$.

In particular, the condition for coexistence $\left(A_{1}, A_{2}<1\right)$ becomes

$A_{21}<\frac{1-1 / k_{2}}{1-1 / k_{1}}<\frac{1}{A_{12}}$

whereas the condition for founder control or bistability $\left(A_{1}, A_{2}>1\right)$ is simply the reverse. Hence, we see that coexistence is possible if $A_{12} A_{21}<1$, whereas founder control may arise if $A_{12} A_{21}>1$.

In Table 2, we summarize the conditions for obtaining the different competition outcomes when unfavourable patches are sinks. We again focus on patch occupancy as determined by effective preferences.

\section{Discussion}

Biological invasions are occurring more and more frequently, and they have the potential to permanently alter ecosystems, for example by removing native species. Accordingly, there is enormous recent interest in empirical and theoretical investigations of invasion processes (Hastings et al. 2005; Taylor and Hastings 2005). A typical first modelling approach considers a single species (the potential invader) in a homogeneous landscape and aims to understand the mechanisms behind reproduction and dispersal to estimate spread. Realistic invasion processes, however, happen in the presence of other interacting species and in heterogeneous landscapes. The combination of these two aspects leads to new opportunities for the potential invader and the species it interacts with. For example, the 'environmental heterogeneity hypothesis of invasions' speculates that heterogeneity could both increase the success of invading species and decrease the impact on native species by promoting coexistence mechanisms that are not present in homogeneous landscapes (Melbourne et al. 2007). The main motivation for our work was to study movement-based mechanisms that could support or dispel this hypothesis or, more generally, affect the invasion process in a heterogeneous landscape.

Table 2 Summary of outcomes when unfavourable patches are sinks

\begin{tabular}{ll}
\hline Outcome & Conditions \\
\hline Stronger competitor wins & Stronger (weaker) competitor has sufficiently large (small) effective preference \\
Weaker competitor wins & Stronger (weaker) competitor has sufficiently small (large) effective preference \\
Coexistence & Effective preference is similar; inferior competitor occupies favourable patches more efficiently; competition is weak \\
Founder control & Same as for 'coexistence', but competition is strong \\
\hline
\end{tabular}


Since the work by Fisher (1937), reaction-diffusion equations are often the framework of choice to model biological invasions, most frequently as single-species models. Several consumer-resource/predator-prey models in homogeneous environments exist, see Lewis et al. (2016) and references therein, but only a few treat competitive interactions (Okubo et al. 1989; Lewis et al. 2002). Models for population spread in fragmented landscapes were formulated by Shigesada et al. (1986) and then extended to include a detailed description of movement behaviour at interfaces between habitat types (Maciel and Lutscher 2013, 2015). These are almost exclusively single-species models with only a few exceptions (Pacala and Roughgarden 1982; Cruywagen et al. 1996). In our current work, we studied how the patchtype-dependent movement behaviour affects and alters the interaction of two competing species in a periodic, patchy environment.

We considered two combinations of patch types in an infinite, periodically alternating landscape: SF/MF, where both patch types are population dynamic sources that differ in carrying capacity, and SF/U with sources and sinks. To focus on movement behaviour, we chose the two species to be as similar as possible otherwise, i.e. in terms of growth rates and carrying capacities. We chose interaction parameters in such a way that the same species won the local competition in each patch type. We then illustrated that movement behaviour, encoded by the single dimensionless parameter $k_{i}$ in (10), the 'effective patch preference', can change the outcome of competition to coexistence, to bistability, and to a reversal of competitive dominance. The two scenarios revealed two different insights. In the $\mathrm{SF} / \mathrm{U}$ scenario, high effective patch preference is beneficial for species persistence. Since the lesser-quality patches are sinks, individuals should enter these at a low rate but move through them at high speed. This negative relationship between probability to enter a patch of certain habitat quality and the movement rate inside the patch has been confirmed empirically for a number of species, in particular for habitat specialists (Kuefler et al. 2010). On the other hand, in SF/MF environments, intermediate effective patch preference seems to be best for species persistence. Very high preference values in the competitively superior species can lead to a reversal of the competitive outcome and favour the competitively inferior species. Our next step in this direction is to study the evolution of patch preference and dispersal in an adaptive dynamics setting (Maciel et al. 2018).

Questions of population persistence and competitive outcomes provide long-term information, but in the management of invasive species, short-term predictions of spread are often more important. We therefore studied the predicted rates of spatial spread of one species into its competitor at steady state. The insights here are somewhat more subtle. For example, a high probability of moving into a good patch $\left(\alpha_{i}\right)$ enhances population persistence but can slow its spread since individuals tend to stay in a good patch and not move on. A higher diffusion rate in $\mathrm{MF}$ patches can slow or even inhibit the spread of a weaker competitor (Fig. 7). Most interesting, however, is the insight that movement characteristics of an inferior resident species can decrease or increase the spread rate of a superior invader indirectly but strongly, for example prohibiting spread. It is therefore clear that biological invasions are more complex than single-species models make us believe and that species interactions need to be taken into account, in particular if landscapes are heterogeneous.

With regard to the "environmental heterogeneity hypothesis' (Melbourne et al. 2007), our investigation shows that both predictions can arise: heterogeneity can lead to higher invasion success and can decrease the negative impacts on resident species. But heterogeneity can do a lot more: depending on how otherwise almost identical species move, the competitive outcome can be reversed or bistability can result.

Red squirrels are being driven to extinction by invasive grey squirrels in much of Britain, and the invasion takes place in a highly heterogeneous environment (Rushton et al. 1997). Red squirrels remain mostly in remote coniferous forests, but as they can sometimes coexist with grey squirrels for decades after the grey squirrels arrive in a location, it is not clear for how much longer. Habitat requirements are somewhat similar for both species in that both prefer coniferous trees, but grey squirrels also use mixed and broadleaved forests (Bryce et al. 2002). Squirrels have home ranges, and a young squirrel often must disperse to find its own range. Squirrels are highly mobile. They can disperse on the order of several home-range sizes, and there is evidence that males can view the landscape as finegrained, so that individuals easily move between fragments (Andrén and Delin 1994). There is also clear evidence that squirrels adjust their movement to landscape features (quality, edges) (Bakker and van Vuren 2004; Haughand and Larsen 2004). Okubo et al. (1989) studied the spread of the grey squirrel in Britain with a spatially homogeneous model. Their estimated diffusion rates, however, take spacing between suitable habitat patches into account. They suggest values between 1 and $20 \mathrm{~km}^{2} /$ year.

For an order of magnitude difference (i.e. $\epsilon=0.1$ ), one would like to consider heterogeneity in terms of patches of 0.1 to $2 \mathrm{~km}^{2}$ or less. Forest inventory and other landscape data are often available on even smaller scales. Many animals, however, have home ranges; for squirrels, these range between 0.01 and $0.1 \mathrm{~km}^{2}$. For scales smaller than these, the pointwise density approach of reaction-diffusion equations and species interactions may not be the appropriate modelling framework any more. Yet, as we demonstrated with our numerical simulations, 
the order of magnitude difference in scales is necessary for the derivation of the homogenized equation but not (necessarily) for it being a surprisingly good approximation to the small-scale equations.

Gurnell et al. (2004) demonstrated negative population dynamic effects of grey on red squirrels on patches of $0.4 \mathrm{~km}^{2}$. The effects include a reduction in recruitment and summer breeding of red squirrels in the presence of grey squirrels. The authors caution that longer-term studies are required because of large annual variation. Okubo et al. (1989) consider the competitive effect of the red squirrel on the grey as very small. According to our results, in regions where favourable (woodlands) and unfavourable (e.g. farmland) habitats are interspersed, the stronger competitor (grey squirrel) will dominate if it has a reasonably strong effective preference (Table 2). Since grey squirrels are also larger than red ones, we speculate that their potential movement rate in unfavourable patches is at least as large as for the red ones, so that they will win the competition (Fig. 8). In regions where more (coniferous) and less (broadleaved) favourable forest patches are interspersed, the stronger competitor only needs to have a similar effective preference to outcompete the inferior species, so that the greys should again win (Table 1). Overall, our work indicates that red squirrels would need to have significantly different effective patch preference (in terms of movement behaviour) to stand a chance against their grey relatives. This is, however, only one tiny piece of the puzzle. There are several other factors (e.g. disease) that contribute to the interaction between red and grey squirrels and to the decline of the red ones.

Our analysis is based on homogenization techniques and multi-scale analysis. These techniques are well developed in general, although the application to models with discontinuous interface conditions is very recent (Alqawasmeh 2017; Yurk and Cobbold 2018). As is often the case, the results from this technique are valid far beyond the formal limit of fine-scale heterogeneity, in which they are derived. Our comparison between the prediction by the homogenized model and the numerical results for the full spatial model confirms this pattern and underlines the value that these techniques can have for theoretical ecology. As mentioned above, care is required when choosing the spatial scales of the model and testing the applicability of homogenization. We pose as a future challenge the development of homogenization techniques on more than two scales: the scale of habitat variation, the scale of population interaction, and the scale of observation.

Previous reaction-diffusion models for competing species in two-type landscapes (Pacala and Roughgarden 1982; Cruywagen et al. 1996) did not incorporate movement behaviour at interfaces and, because of that, may have missed some important relationships (Maciel and Lutscher 2013). However, the work by Cruywagen et al. (1996) in particular used another approximation to find profiles of steady states. Extending their techniques to our more general model is one of the future challenges that results from our work and that may give even more insights into the way in which movement shapes competition in heterogeneous landscapes. A much greater challenge is to extend the existence results for spreading speeds and travelling waves, as well as conditions for linear determinacy, to the full, spatially heterogeneous equations, and to characterize situations better in which the linearization technique could fail. Our work is also related to earlier work on discrete-time integrodifference equations in patchy landscapes, where movement behaviour was independent of patch type, for example for wind-dispersed plants (Samia and Lutscher 2010). Recent developments on the theory of dispersal kernels (Musgrave and Lutscher 2014) will allow us to examine the mechanisms from our work in the context of integrodifference equations more closely.

Our work limits patch preference $(\alpha)$ in movement locally to the interface between patch types. Work by Cantrell et al. (2007) considers habitat quality a continuous variable and uses a taxis term to model movement upwards an environmental gradient. The authors did not consider spatial spread rates, but their results on persistence conditions and outcome of competition are comparable to ours. For example, they found that taxis towards better habitat is helpful for species persistence and that very strong taxis towards favourable sites in one species allows a weaker competing species to coexist by using less favourable sites.

Finally, we remark that we assumed that neither of the two species experiences an Allee effect where the percapita growth rate peaks at intermediate densities. With an Allee effect, the linearization procedure to determine population persistence and spread rates will not work. The homogenization technique can still be applied. In a single-species model, it has already revealed a number of interesting outcomes such as front localization (Maciel and Lutscher 2015). In a non-spatial model for competition and Allee effect, a number of interesting effects were found, for example the scenario that neither species can persist individually but together they can Lutscher and Iljon (2013). Since Allee effects are quite common in nature and might help manage invasions (Tobin et al. 2011), future research should aim to include these features into multi-species models for invasions.

Acknowledgements We thank Brian Yurk and Christina Cobbold for sharing their preprint about homogenization for models with discontinuous densities with us. The detailed comments of two anonymous reviewers have helped us improve our manuscript considerably. GAM gratefully acknowledges a postdoctoral grant from CNPq - Brazil. FL is thankful for a Discovery Grant (RGPIN-201604795) and a Discovery Accelerator Supplement from the National Science and Engineering Research Council of Canada. 


\section{References}

Alqawasmeh Y (2017) Models for persistence and spread of structured populations in patchy landscapes. $\mathrm{PhD}$ thesis, University of Ottawa. https://ruor.uottawa.ca/handle/10393/36845

Andrén H, Delin A (1994) Habitat selection in the Eurasian red squirrel, Sciurus vulgaris, in relation to forest fragmentation. Oikos 70(1):4348

Bakker V, van Vuren D (2004) Gap-crossing decisions by the red squirrel, a forest-dependent small mammal. Conserv Biol 18:689697

van den Bosch F, Hengeveld R, Metz J (1992) Analysing the velocity of animal range expansion. J Biogeogr 19:135-150

Bryce J, Johnson P, Macdonald D (2002) Can niche use in red and grey squirrels offer clues for their apparent coexistence? J Anim Ecol 39:875-887

Cantrell R, Cosner C, Lou Y (2007) Advection-mediated coexistence of competing species. Proceedings of the Royal Society of Edinburgh Section A 137(3):497-518

Crone E, Schultz C (2008) Old models explain new observations of butterfly movement at patch edges. Ecol 89(7):2061-2067

Cruywagen G, Kareiva P, Lewis M, Murray J (1996) Competition in a spatially heterogeneous environment: modelling the risk of spread of a genetically engineered population. Theor Popul Biol 49(1):1-38

Duncan JP, Rozum RN, Powell JA, Kettenring KM (2017) Multi-scale methods predict invasion speeds in variable landscapes. Theor Ecol 10(3):287-303

Fisher R (1937) The advance of advantageous genes. Ann Eugen 7:355-369

Garlick M, Powell J, Hooten M, McFarlane L (2011) Homogenization of large-scale movement models in ecology. Bull Math Biol 73(9): 2088-2108

Garlick MJ, Powell JA, Hooten MB, MacFarlane LR (2014) Homogenization, sex, and differential motility predict spread of chronic wasting disease in mule deer in southern Utah. J Math Biol 69(2):369-399

Gurnell J, Wauters L, Lurz PWW, Tosi G (2004) Alien species and interspecific competition: effects of introduced eastern grey squirrels on red squirrel population dynamics. J Anim Ecol 73:26-35

Hastings A, Cuddington K, Davies K, Dugaw C, Elmendorf A, Freestone A, Harrison S, Holland M, Lambrinos J, Malvadkar U, Melbourne B, Moore K, Taylor C, Thomson D (2005) The spatial spread of invasions: new developments in theory and evidence. Ecol Lett 8:91-101

Haughand D, Larsen K (2004) Exploration correlates with settlement: red squirrel dispersal in contrasting habitats. J Anim Ecol 73: 1024-1034

Hosono Y (1998) The minimal speed of traveling fronts for a diffusive lotka volterra competition model. Bull Math Biol 60:435-458

Kuefler D, Hudgens B, Haddad N, Morris W, Hurgate N (2010) The conflicting role of matrix habitats as conduits and barriers for dispersal. Ecol 91(4):944-950

Lewis M, Li B, Weinberger H (2002) Spreading speed and linear determinacy for two-species competition models. J Math Biol 45:219-233

Lewis M, Petrovskii S, Potts J (2016) The mathematics behind biological invasions, 1st edn. Springer, Berlin

Ludwig D, Aronson D, Weinberger H (1979) Spatial patterning of the spruce budworm. J Math Biol 8:217-258

Lutscher F, Iljon T (2013) Competition, facilitation and the Allee effect. Oikos 122:621-631

Lutscher F, Lewis M, McCauley E (2006) Effects of heterogeneity on spread and persistence in rivers. Bull Math Biol 80(3):752760
Maciel GA, Lutscher F (2013) How individual movement response to habitat edges affects population persistence and spatial spread. Am Nat 182(1):42-52

Maciel GA, Lutscher F (2015) Allee effects and population spread in patchy landscapes. J Biol Dyn 9(1):109-123

Maciel GA, Cosner C, Cantrell R, Lutscher F (2018) (in prep) Evolutionarily stable movement strategies in reaction-diffusion models with edge behavior

Melbourne BA, Cornell HV, Davies KF, Dugaw CJ, Elmendorf S, Freestone AL, Hall RJ, Harrison S, Hastings A, Holland M, Holyoak M, Lambrinos J, Moore K, Yokomizo H (2007) Invasion in a heterogeneous world: resistance, coexistence or hostile takeover? Ecol Lett 10:77-94

Musgrave J, Lutscher F (2014) Integrodifference equations in patchy landscapes. I. dispersal kernels. J Math Biol 69:583-615

Okubo A, Maini PK, Williamson MH, Murray JD (1989) On the spatial spread of the grey squirrel in Britain. Proc R Soc Lond B Biol Sci 238(1291):113-125

Othmer H (1983) A continuum model for coupled cells. J Math Biol 17:351-369

Ovaskainen O, Cornell SJ (2003) Biased movement at a boundary and conditional occupancy times for diffusion processes. J Appl Probab 40:557-580

Pacala S, Roughgarden J (1982) Spatial heterogeneity and interspecific competition. Theor Popul Biol 21:92-113

Pavliotis G, Stuart A (2008) Multiscale methods: averaging and homogenization. Springer, New York, USA

Powell J, Zimmermann N (2004) Multiscale analysis of active seed dispersal contributed to resolving Reid's paradox. Ecol 85(2):490-506

Reeve J, Cronin J, Haynes K (2008) Diffusion models for animals in complex landscapes: incorporating heterogeneity among substrates, individuals and edge behaviours. J Anim Ecol 77:898-904

Rushton S, Lurz PWW, Fuller R, Garson P (1997) Modelling the distribution of the red and grey squirrel at the landscape scale: a combined GIS and population dynamics approach. J Appl Ecol 34(5):1137-1154

Samia Y, Lutscher F (2010) Coexistence and spread of competitors in heterogeneous landscapes. Bull Math Biol 72(8):2089-2112

Schtickzelle N, Baguette M (2003) Behavioural responses to habitat patch boundaries restrict dispersal and generate emigrationpatch area relationships in fragmented landscapes. J Anim Ecol 72(4):533-545

Schultz C, Crone E (2001) Edge-mediated dispersal behavior in a prairie butterfly. Ecol 82(7):1879-1892

Shigesada N, Kawasaki K, Teramoto E (1986) Traveling periodic waves in heterogeneous environments. Theor Popul Biol 30:143-160

Skellam J (1951) Random dispersal in theoretical populations. Biometrika 38:196-218

Strikwerda JC (2004) Finite difference schemes and partial differential equations, 2nd edn. Society for Industrial and Applied Mathematics, Philadelphia

Taylor C, Hastings A (2005) Allee effects in biological invasions. Ecol Lett 8:895-908

Tobin PC, Berec L, Liebhold AM (2011) Exploiting Allee effects for managing biological invasions. Ecol Lett 14:615-624

Turchin P (1998) Quantitative analysis of movement: measuring and modeling population redistribution in plants and animals. Sinauer, Sunderland, Massachusetts, USA

Weinberger H (1982) Long-time behavior of a class of biological models. SIAM J Math Anal 13:353-396

Weinberger HF (2002) On spreading speeds and traveling waves for growth and migration models in a periodic habitat. J Math Biol 45:511-548

Yurk B, Cobbold C (2018) Homogenization techniques for population dynamics in strongly heterogeneous landscapes. J Biol Dyn 12:171-193 